\title{
An Energy SAVing Algorithm to Prolong THE LifETIME OF WiRELESS SENSOR NETWORK
}

\author{
Monika Raghatate $^{1}$ and Prof. Dipak W. Wajgi ${ }^{2}$ \\ ${ }^{1}$ Department of Computer Engineering, St. Vincent Pallotti College of \\ Engineering and Technology, Nagpur, India \\ ${ }^{2}$ Department of Computer Engineering, St. Vincent Pallotti College of \\ Engineering and Technology, Nagpur, India
}

\begin{abstract}
This paper considers a heterogeneous network of energy constrained sensors deployed over a region. Each Normal sensor node in a network is systematically gathering and transmitting sensed data to the clusterhead, and then cluster head sending data to a base station (via intermediate cluster- heads). This paper focuses on reducing the energy consumption and hence improving lifetime of wireless sensor Networks. Clustering sensor node is an effective topology for the energy constrained networks. So energy saving algorithm has been developed in which clusters are formed considering a subset of high energy nodes as a cluster-head and another subset of powerful nodes is ask to go to sleep. When Cluster heads deplete their energy another subset of nodes becomes active and acts as a cluster head. Proposed approach is implemented in MATLAB, Simulation results shows that it can prolong the network lifetime than LEACH protocol, and achieves better performance than the existing clustering algorithms such as LEACH.
\end{abstract}

\section{KEYWORDS}

Cluster Head, Energy Saving Algorithm, MATLAB, Network Lifetime, Wireless Sensor Network.

\section{INTRODUCTION}

A wireless sensor networks (WSN) is a network of small devices called as sensor nodes deployed over a geographical area for monitoring physical phenomena like seismic events, vibrations, temperature, humidity and so on [1]. Typically, a sensor node is a small/tiny device which has three basic components: sensing subsystem, processing subsystem and wireless communication subsystem. A sensing subsystem for sensing data from the physical surrounding environment, a processing subsystem for processing data and storage, and a wireless communication subsystem for data transmission. It also has a power source often consists of a battery which has limited energy budget and it is inconvenient or impractical to recharge the battery or nodes may be deployed in an environment where human being cannot reach. For example, in underwater scenario, there is no plugin socket to provide power as per requirement.

Military applications motivated the development of wireless sensor network. However, Now in many application areas including environment, Hospitals, agriculture, habitat monitoring etc. wireless sensor networks are used, due to various limitations arising from their inexpensive nature, weight, limited size, and ad hoc method of deployment; To prolong the lifetime of the network it is necessary to find energy efficient route setup and reliable transmission of data from the sensor nodes to the base station or sink [2]. To improve the performance of the sensor network application, it is necessary to improve the lifetime of network and it can be possible by saving energy of nodes. In some cases it is possible to gather energy from the external environment [3].

DOI : 10.5121/ijwmn.2014.6503 
However, external power supply sources often show an interrupted behavior so that an energy buffer is needed as well. Energy is a very limited resource and must be used properly. Therefore, energy conservation is a fundamental issue in the design of systems based on wireless sensor networks.

The remainder of the paper is organized as follows. In Section II, review of literature is discussed. Section III describes Mathematical Model. Section IV describes Proposed Approach. In Section V simulation Results are discussed. Finally, Section VI gives concluding remarks and future scope.

\section{RELATED WORK}

\subsection{Leach}

Low Energy Adaptive Clustering Hierarchy (LEACH) protocol [4] has proposed by W. R. Heinzelman, A. P. Chandrakasan and H. Balakrishnan. It is one of the most popular hierarchical routing algorithms for sensor networks. The idea is to form clusters of the sensor nodes based on the received signal strength and use local cluster heads as routers to the sink. This reduces energy consumption since the transmissions will only be done by such cluster heads rather than all sensor nodes. Minimum number of cluster heads (CHs) is estimated to be 5\% of the total number of nodes. All the data processing such as data fusion and aggregation are local to the cluster. Cluster heads change randomly over time in order to balance the energy depletion of nodes. The working of LEACH is divided into two phases: Set up Phase and steady state phase. Each round starts with a set-up (clustering) phase, where clusters are formed, followed by a steady-state (transmission) phase in which data packets are transferred from normal nodes to cluster heads. After data aggregation, cluster heads will transmit the aggregated data to the Base Station.

\subsubsection{Disadvantages}

Homogeneous distribution of sensor nodes is assumed.

LEACH is not applicable in large regions.

Distribution of the $\mathrm{CH}$ nodes in the network is not uniformed.

\subsection{PEGASIS Protocol}

S. Lindsey and C. Raghavendra [5] have proposed Power Efficient Gathering in Sensor Information Systems (PEGASIS) protocol. It is an improved version of LEACH. Rather than forming clusters, it forms chains of sensor nodes. Single node is responsible for transferring the aggregated data to the base station. Each node aggregates the collected data with its own data, and then passes the aggregated data to the next ring. PEGASIS is different from LEACH because it employ multi hop transmission and select only one node to transmit the data to the sink or base station. Since it eliminates the overhead caused by dynamic cluster formation, multi hop transmission and data aggregation is employed; PEGASIS achieves better performance than LEACH. However for distant nodes excessive delay is introduced, especially for large networks and single leader can be a bottleneck.

\subsection{TEEN Protocol}

Threshold sensitive Energy Efficient sensor Network Protocol (TEEN) [6] has proposed by A. Manjeshwar and D. P. Agarwal. In this protocol clusters are formed considering the nearer nodes, with a cluster heads to transmit the collected data to one upper layer. After forming the clusters, 
cluster heads broadcast two threshold values. First one is hard threshold; it is minimum possible value of an attribute to trigger a sensor node. Hard threshold allows nodes transmit the event, if the event occurs in the range of interest. Therefore a significant reduction of the transmission delay occurs. Unless a change of minimum soft threshold occurs, the nodes don't send a new data packet. Employing soft threshold prevents from the redundant data transmission. Since the protocol is to be responsive to the sudden changes in the sensed attribute, it is suitable for timecritical applications. They have also proposed Adaptive Threshold sensitive Energy Efficient sensor Network Protocol (APTEEN) protocol [7]. The protocol is an extension of TEEN aiming to capture both time-critical events and periodic data collections. The network architecture is same as TEEN. After forming clusters the cluster heads broadcast attributes, the threshold values, and the transmission schedule to all nodes. Cluster heads are also responsible for data aggregation in order to decrease the size data transmitted so energy consumed. According to energy dissipation and network lifetime, TEEN gives better performance than LEACH and APTEEN because of the decreased number of transmissions. The main drawbacks of TEEN and APTEEN are overhead and complexity of forming clusters in multiple levels, implementing threshold-based functions and dealing with attribute based naming of queries.

\subsection{SEP Protocol}

Stable Election Protocol (SEP) protocol [8] has proposed by G. Smaragdakis, I. Matta and A. Bestavros. This protocol is an extension to the LEACH protocol. It is a heterogeneous aware protocol which is based on weighted election probabilities of each node to become cluster head according to their respective energy. SEP ensures that the cluster head election is randomly selected and distributed based on the fraction of energy of each node considering a uniform use of the nodes energy. In SEP, two types of nodes i.e. two tier in-clustering and two level hierarchies were considered.

\subsection{DEEC Protocol}

Distributed Energy Efficient Clustering Protocol (DEEC) [9] has proposed by Q. Li, Z. Qingxin and W. Mingwen. This protocol is a cluster based scheme for two level and multi level energy heterogeneous wireless sensor networks. This scheme, selects cluster heads using the probability based on the ratio between residual energy of each node and the average energy of the network. The epochs are different according to their initial and residual energy of being cluster-heads for nodes. The chance of the becoming cluster heads depends on nodes initial and residual energy, the nodes with high initial and residual energy have more chances than the nodes with low energy.

\subsection{HEED Protocol}

Hybrid Energy Efficient Distributed clustering Protocol (HEED) [10] has proposed by O. Younis and S. Fahmy. The basic scheme of LEACH is extended by HEED using residual energy as primary parameter and network topology features for e.g. node degree, distances to neighbours etc. are used as a secondary parameters to break tie between candidate cluster heads, as a metric for cluster selection to achieve power balancing[13]. The process of clustering is divided into a number of iterations, and in each iterations, nodes which are not covered by any cluster head shows their probability of becoming a cluster head. Since these energy-efficient clustering protocols enable every node to independently and probabilistically decide on its role in the clustered network, they cannot guarantee minimum elected set of cluster heads $[12,13]$. 
International Journal of Wireless \& Mobile Networks (IJWMN) Vol. 6, No. 5, October 2014

\subsection{EEICCP Protocol}

Shalli Rani et.al has proposed Energy Efficient inter cluster coordination Protocol [11] for the wireless sensor networks, It is a cluster based algorithm in which different levels of clusters are considered on the basis of received signal strength to recognize the distance of the clusters from the BS (base station) and to determine the number of cluster coordinators to make routes for the $\mathrm{CHs}$ to transmit the data. The protocol depends upon the fact that some cluster head sends data directly to the base station (Single hop) and the some send by multi hop transmission, but energy consumption in conventional protocols increases due to multi path fading channel which affects the network life time. An attempt has been made to reduce this power loss in to free space model that is $\mathrm{d} 2$. The arrangements of the nodes has been done in this way that one cluster always, is very close to the base station i.e. in line of sight propagation and that cluster will have head nodes sufficient for all the below clusters which will forward the data of all those clusters. Layers of clusters have been so there is always one cluster coordinator for every lowest cluster. The division of clusters is done from top to bottom.

EEICCP protocol works by starting the election phase in which the cluster heads are elected according to the distance based on RSS. Number of clusters is fixed so as the cluster heads and the cluster coordinators after election of cluster coordinators by the $\mathrm{CHs}$, a cluster id is assigned to each cluster head and the cluster coordinator. This id is transmitted by each cluster to their nodes by the advertisement message. And cluster co-coordinators also pass their own ids. After that the transmission phase begins in which data is transferred to the cluster head and that data is passed to the base station with the help of CCOs. In first round the data is collected by the $\mathrm{CH}$ of that cluster which has data to send, then in the other iterations the data is passed to the base station with the help of cluster co-coordinators.

EEICCP outperforms conventional protocols that send data directly to the BS through their respective CHs. Dividing the network into layers of clusters has been proved to be a good approach in reducing the energy to a great extent. Each node has the equal responsibility of receiving data from all other nodes in the cluster and to transmit the aggregating signal to the base station. Simulations show that EEICCP reduces start energy 151 times than both HCR and LEACH, energy of one iteration by $0.2926 \times 10^{4}$ times than HCR and $0.3169 \times 10^{4}$ times than LEACH. Thus total energy reduction is $43 \%$ than HCR and 50\% than LEACH.

\section{Mathematical Model}

Many of the research protocols have used the first order radio model as described in [5]. Energy is dissipated while transmitting and receiving the data and energy consumption for the short distance is $d 2$ when propagation is in line of sight and $d 4$ for the long distance due to multipath fading propagation [1].

Transmission energy and receiving energy are calculated as follows:

$$
\begin{aligned}
& E_{T x}(l, d)=\left\{\begin{array}{c}
l E_{\text {elect }}+l E_{f s} d^{2}, d<d o \\
l E_{\text {elect }}+l E_{m p} d^{4}, d>d o
\end{array}\right. \\
& E_{R x}(L)=E_{\text {elect }} L
\end{aligned}
$$


Where $\mathrm{d}$ is a difference between transmitter and receiver. And $\mathrm{L}$ is a the length of the message in bits,

$$
\mathrm{d}_{0}=\sqrt{ } \mathrm{E}_{\mathrm{fs}} / \mathrm{E}_{\mathrm{mp}}
$$

In proposed approach, two types of sensor nodes, i.e., the High Energy nodes (powerful nodes) and normal nodes are considered. Let us assume that there are ' $\mathrm{N}$ ' numbers of sensor nodes deployed in an area, where the initial energy of the normal nodes is assumed to be $E_{0}$, and $m$ is the percentage of powerful nodes, which own $a$ times more energy than the normal ones. Thus $(m *$ $N)$ powerful nodes are equipped with initial energy of $($ Eo $*(1+\alpha))$, and $((1-\mathrm{m}) * \mathrm{~N})$ normal nodes are equipped with initial energy of $E O$. The total initial energy of the network is given by:

$$
\begin{aligned}
\text { Etotal } & =N \cdot(1-m) \cdot E o+N \cdot m \cdot E o \cdot(1+\alpha) \\
& =N \cdot E o \cdot(1+\alpha \cdot m)
\end{aligned}
$$

So, by using heterogeneous network the total energy of the system is increased by a factor of $(1+\alpha$ $\cdot \mathrm{m})$.

\section{Proposed Approach}

According to the proposed approach a heterogeneous network with the sensor nodes having different energy levels and processing power is assumed. Some high Energy nodes (Powerful nodes/Higher nodes) having $a$ times more energy than the normal nodes are deployed in the network. A Subset of Powerful node from the set of higher nodes is selected as a $\mathrm{CH}$ based on the fact that they should not be in a communication range of each other. Another subset of higher nodes is asked to go to sleep. The subset of Powerful node which is chosen as a $\mathrm{CH}$ broadcast its presence to all normal sensor nodes and starts receiving data from the sensors which are members of their cluster. Normal sensor decides to which cluster they want to belong based on the signal from the broadcast. It is assumed that the stronger the signal, the closer the head is and therefore the head with strongest signal are chosen. All the cluster members send the sensed data to the $\mathrm{CH}$. The $\mathrm{CH}$ will send the aggregated data to the Base Station directly or by using some intermediate $\mathrm{CH}$.

When the energy of the $\mathrm{CH}$ reaches to zero, another subset of nodes would act as a Cluster Head. This information about the new $\mathrm{CH}$ will be sent to all normal nodes and the same process is repeated. The network will be stable or dead when the energy of all cluster heads becomes zero.

\subsection{Algorithm}

The algorithm is divided into two phases

\subsubsection{Selection Phase}

Step1: Select a Subset of high energy node as cluster head.

Step2: Cluster Head broadcast a hello message to all normal sensor nodes and starts receiving data from the sensors that have decided to become a cluster member.

Step3: If the Cluster Head energy is depleted completely or reaches to the threshold value i.e. zero, then high energy nodes of another subset will act as Cluster Head \& Repeat step 2.

In selection phase a subset of high energy node is selected as a cluster head and other subset of node is asked to go to sleep. The cluster head sends an advertisement message to the normal sensor nodes and the nodes send acknowledge message to the cluster head. Normal sensors decide 
to which cluster they wish to belong based on the distance of node from cluster head. Cluster head collects data from all the members of their cluster and send it to base station. When the energy of cluster head becomes zero then other subset of high energy node will acts as a cluster head as same process is repeated.

\subsubsection{Data Transfer Phase}

Step1: The cluster members send the data to the cluster head.

Step2: Cluster Head will collect the data from all the members of their cluster.

Step3: Cluster Head will transmit the aggregated data to base station through the cluster heads nearer to it.

Transmission begins from the depth first $\mathrm{CH}$ which goes to the Cluster head of the next higher level cluster and so on as shown in Figure 1 which is finally sent to the BS by the $\mathrm{CH}$ of the last cluster nearest to the BS. In this way the distance travelled is decreased, and energy is saved because more energy is require to send data to the base station, if it is located far away from it.

\subsection{Algorithm for Data Transmission}

1. Set $\mathrm{k}=0, \mathrm{n}=1$

2. Set $\mathrm{j}=$ number of clusters $-\mathrm{k}$

3. Repeat while $\mathrm{j}>=1$

4. If $\mathrm{j} !=1$ then Cluster $[\mathrm{j}-1][\mathrm{n}]=$ cluster$[\mathrm{j}][\mathrm{n}]$

Else

Base station $=$ cluster[j][n] .

End of if structure

5. [Decrement] set $\mathrm{j}=\mathrm{j}-1$

Go to step 2

6. End of Step 3 loop

7. Exit 


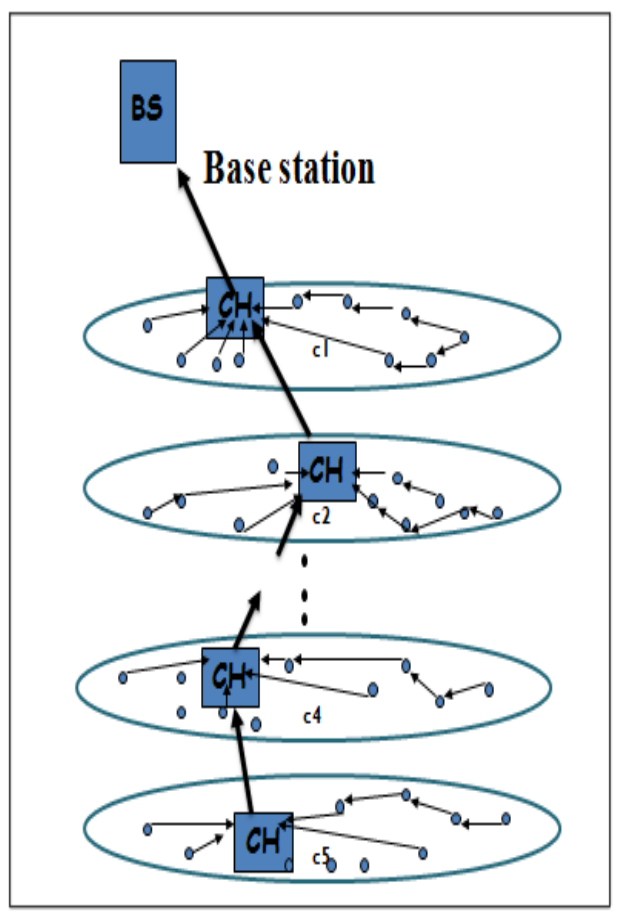

Figure 1. Transfers of Data

\section{Simulation Results AND Discussion}

Based on the analytical work described in Section 4 extensive simulations are carried out in MATLAB. Heterogeneous sensor network of 50 sensor nodes is considered. 44 normal nodes are randomly distributed whereas 6 powerful nodes are deployed deterministically in the $100 \mathrm{~m} * 100 \mathrm{~m}$ area. The base station is located at $(50,130)$. Table 1 . Shows The Simulation parameter. 
Table 1. Simulation Parameters

\begin{tabular}{|l|c|}
\hline \multicolumn{1}{|c|}{ Parameters } & Values \\
\hline Sink & At $(50,130)$ \\
\hline Cluster Radius & $25 \mathrm{~m}$ \\
\hline $\begin{array}{l}E_{\text {elect }} \text { (Energy consumed in the electronics circuit to } \\
\text { transmit or receive the Signal) }\end{array}$ & $50 \mathrm{~nJ} / \mathrm{bit}$ \\
\hline $\begin{array}{l}E_{f s}(\text { Energy consumed by the amplifier to transmit at } \\
\text { a short distance) }\end{array}$ & $10 \mathrm{pJ} / \mathrm{bit} / \mathrm{m} 2$ \\
\hline $\begin{array}{l}E_{m p} \text { (Energy consumed by the amplifier to transmit } \\
\text { at a longer distance) }\end{array}$ & $0.0013 \mathrm{pJ} / \mathrm{bit} / \mathrm{m} 4$ \\
\hline EDA (Data Aggregation Energy) & $5 \mathrm{~nJ} / \mathrm{bit} / \mathrm{signal}$ \\
\hline Message Size & $4000 \mathrm{bits}$ \\
\hline E0, Initial Energy & $0.5 \mathrm{~J}$ \\
\hline
\end{tabular}

Figure 2 shows the deployment of nodes in the network, Blue colour nodes are normal nodes which are deployed randomly and the nodes shown in red colour are high energetic nodes which are deployed by using deterministic approach. The network of $\mathrm{N}$ nodes is considered from which $\mathrm{m}$ percent of nodes are high energetic nodes and remaining nodes are normal nodes, Therefore the high energetic nodes would be $\mathrm{m} * \mathrm{~N}$ and normal nodes would be $(1-\mathrm{m}) * \mathrm{~N}$. For example if we consider the network of 50 nodes i.e. $\mathrm{N}=50$ and $12 \%$ of high energetic nodes i.e. $\mathrm{m}=0.12$ then normal nodes would be $(1-0.12) * 50=44$ and high energetic nodes would be $0.12 * 50=6$.

Base station is located far away from the nodes, as shown in Figure 2. It is located at $(50,130)$. All cluster heads handover their aggregated data to base station for processing and decision making therefore deployment of base station is very important in wireless sensor network 
International Journal of Wireless \& Mobile Networks (IJWMN) Vol. 6, No. 5, October 2014

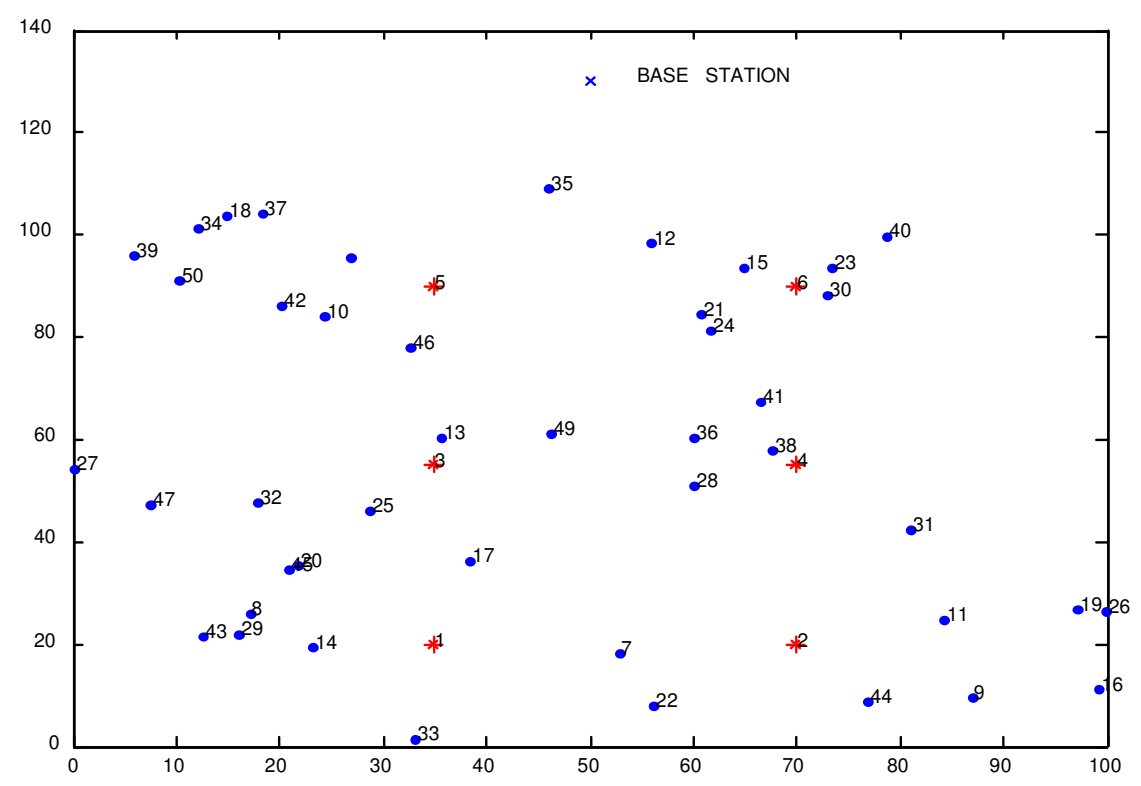

Figure 2. Random Deployment of Normal Nodes

Figure 3 Shows the Cluster Formation considering the subset of high Energy Nodes as a Cluster Head. From the high energy nodes a subset of high energy node is considered as a cluster head e.g. node $1,4,5$ as shown in figure and other subset of node will go to sleep for some duration.

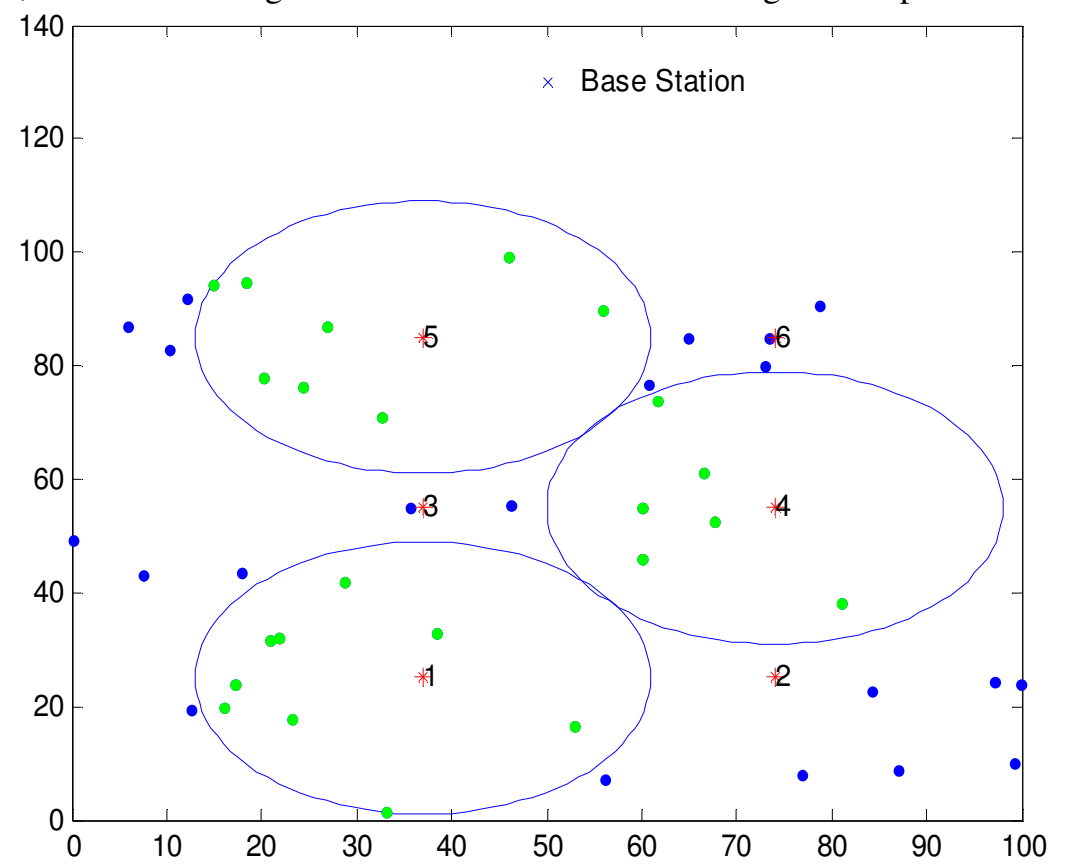

Figure 3. Cluster Formation Considering the First Subset of Nodes as a cluster Head

These cluster head will decide their communication range and form clusters by considering the normal nodes as a cluster members which are in the communication range of that cluster head. Initially round starts from cluster head 1 , it will collect data from the members of their cluster and 
send it to cluster head which is nearer to it i.e. cluster head 4, then cluster head 4 will transfer it to cluster head 5 and finally cluster head 5 will send it to base station.

Similarly second cluster head i.e. cluster head 4 collect data from all cluster member and send it to base station, then in next round cluster head 5 will send gathered data to base station directly. The same process is repeated for number of rounds.

As shown in figure 4, when the energy of cluster heads becomes zero a other subset of higher nodes becomes active and will act as a cluster head and the same process is repeated till the energy of all cluster head becomes zero.

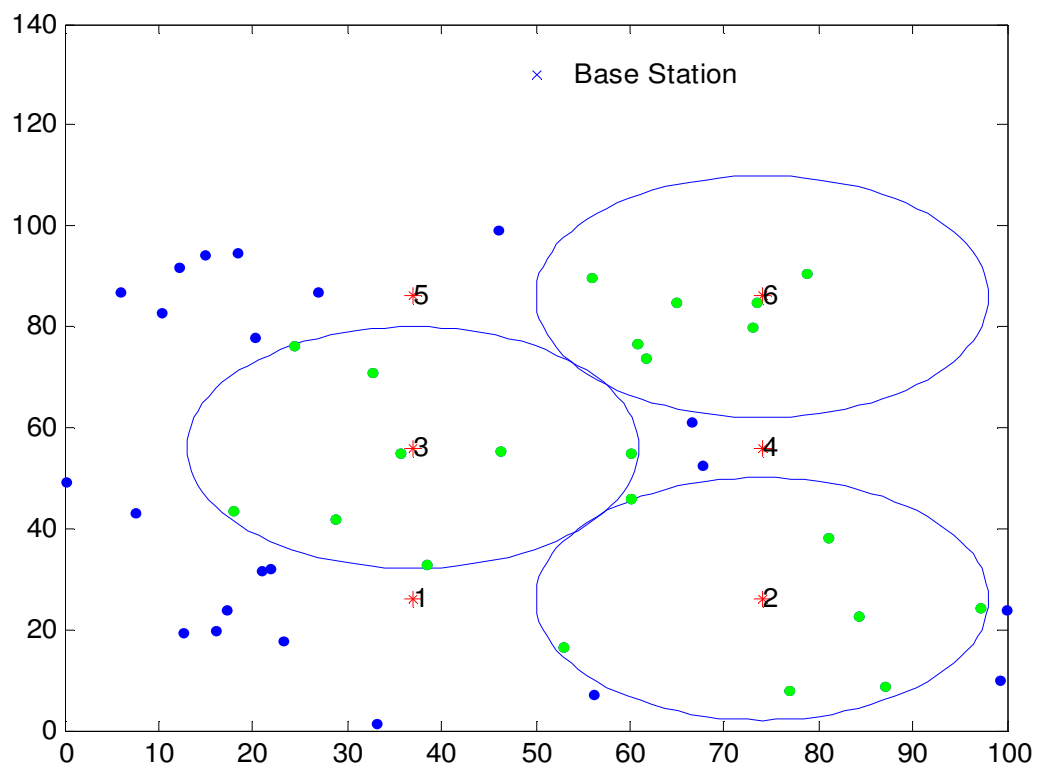

Figure 4. Cluster Formation Considering the Second Subset of Nodes as a cluster Head

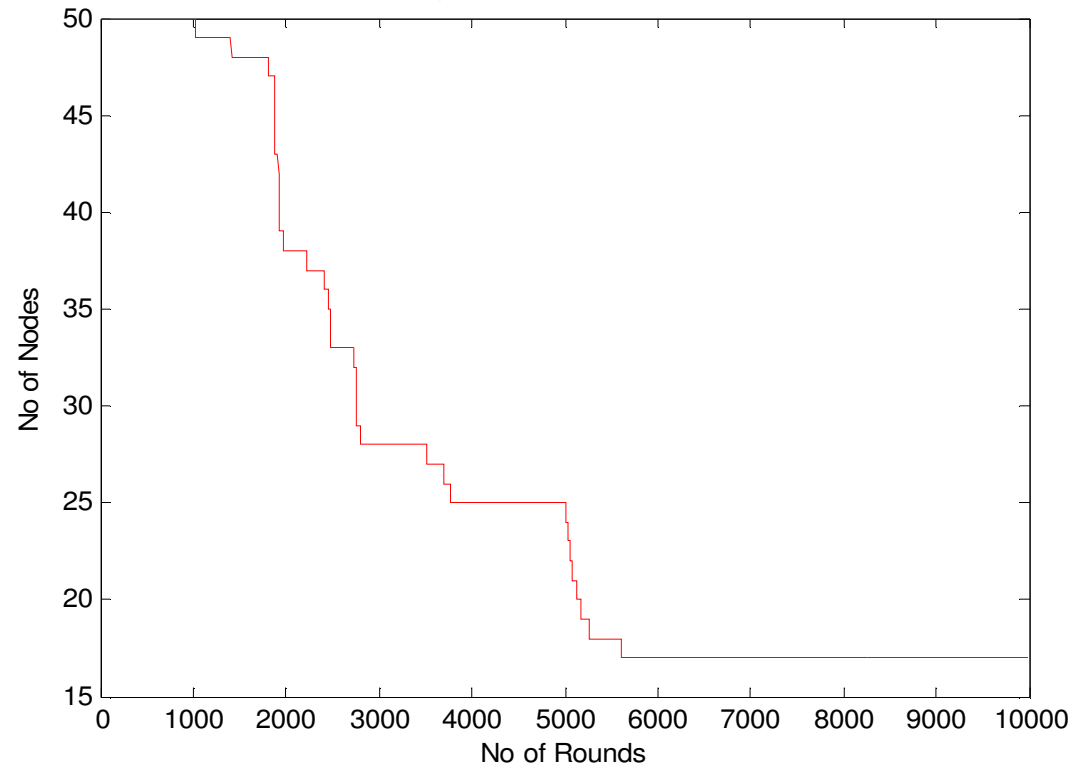

Figure 5. Performance graph of Proposed Approach, showing the number of nodes on y-axis and the rounds on the $\mathrm{x}$-axis 
Figure 5. illustrates the detailed view of the behavior of proposed Approach; Graph shows the number of alive nodes per round where number of rounds are shown on $\mathrm{x}$ axis and no of nodes on $y$ axis . In this case 10000 rounds and 50 nodes are considered. It is observed that 50 nodes remains in network for 1000 rounds and the death of first node occurs after 1000 rounds.

As the numbers of rounds are increases the number of alive nodes decreases and at round 5806 network become dead because energy of all cluster head becomes zero. Here the cluster heads which are nearer to base station depleted their energy faster than other cluster heads because along with their own data it has to send data of other cluster heads.

Figure 6. illustrates a detailed view of the behaviour of proposed approach and LEACH protocol; it shows the number of alive nodes per round. The number of nodes die in Proposed Approach is much less than LEACH Protocol over the same number of rounds.

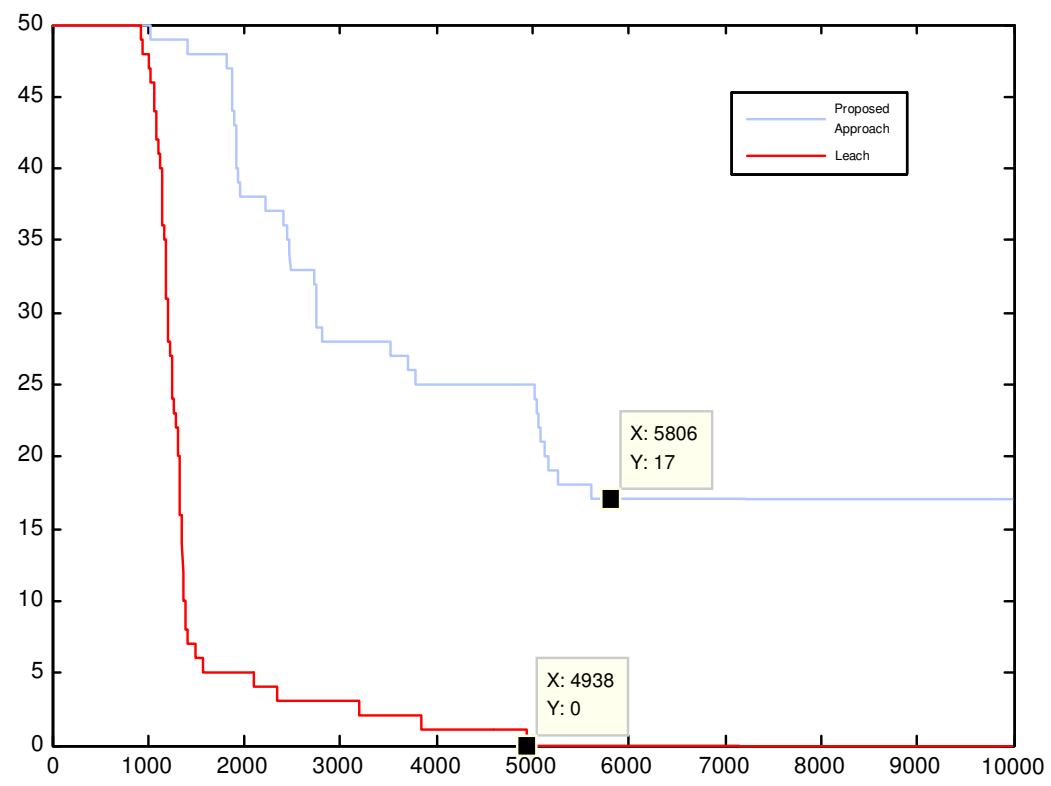

Figure 6. Comparative graphs of Proposed Approach and LEACH Protocol

In proposed Approach death of first node occurs much later than the death of first node in case of LEACH Protocol, and the network works for more number of rounds i.e. 5806 whereas in LEACH it works for only 4938 rounds. From this it can be say that proposed approach prolongs lifetime and shows better performance than LEACH protocol.

\section{Conclusion}

In this paper, a new approach for energy saving and hence prolonging network lifetime in the wireless sensor network has proposed. Algorithms for cluster head selection and data Transmission in wireless sensor network are proposed and implemented. From the simulation results we can say that proposed approach increase network overall energy, saves energy, prolongs lifetime and shows higher performance than LEACH protocol. But the drawback of proposed approach is that when all cluster head dies the network become stable (dead), therefore all sensor nodes are not utililized. Due to this the network does not work till the death of last node.

In future, we planned to design a new approach which will overcome this drawback, and all sensor nodes would be utilized \& network will work until the death of last node. 
International Journal of Wireless \& Mobile Networks (IJWMN) Vol. 6, No. 5, October 2014

\section{REFERENCES}

[1] M. Ye, C. Li, G. Chen, J. Wu., (2005) "EECS: an energy efficient cluster scheme in wireless sensor networks", In IEEE International Workshop on Strategies for Energy Efficiency in Ad Hoc and Sensor Networks (IEEE IWSEEASN-2005), Phoenix, Arizona, April 7-9. .

[2] Sajid Hussain et al., (2005) "Hierarchical cluster-based routing in wireless sensor network", ,jodrey school of computer science acadia university wolfville, pp. 1-33

[3] Zahra Rezaei, Shima Mobininejad, (2012) "Energy Saving in Wireless Sensor Networks", International Journal of Computer Science \& Engineering Survey (IJCSES) Vol.3, No.1.

[4] W. Heinzelman, A. Chandrakasan and H. Balakrishnan, (2000) "Energy-Efficient Communication Protocol for Wireless Microsensor NetworkS", Proceedings of the $33^{\text {rd }}$ Hawaii International Conference on System Sciences (HICSS '00).

[5] S. Lindsey, C. Raghavendra, (2002) "PEGASIS: Power- Efficient Gathering in Sensor Information Systems", IEEE Aerospace Conference Proceedings, Vol. 3, 9-16 pp. 1125- 1130

[6] A. Manjeshwar and D. P. Agarwal, (2001) "TEEN: a routing protocol for enhanced efficiency in wireless sensor networks”, In 1st International Workshop on Parallel and Distributed Computing Issues in Wireless Networks and Mobile Computing

[7] A. Manjeshwar and D. P. Agarwal, (2002) "APTEEN: A hybrid protocol for efficient routing and comprehensive information retrieval in wireless sensor networks", Parallel and Distributed Processing Symposium, Proceedings International, IPDPS, pp. 195-202

[8] G. Smaragdakis, I. Matta, A. Bestavros, (2004) "SEP: A Stable Election Protocol for clustered heterogeneous wireless sensor networks", In Second Internatiolnal Workshop on Sensor and Actor Network Protocols and Applications (SANPA).

[9] Q. Li, Z. Qingxin, and W. Mingwen, (2006) "Design of a distributed energy efficient clustering algorithm for heterogeneous wireless sensor networks", Computer Communications, vol. 29, pp. 2230-7.

[10] Ossama Younis and Sonia Fahmy, (2004) "Distributed Clustering in Ad-hoc Sensor Networks: A Hybrid, Energy-Efficient Approach", In Proceedings of IEEE INFOCOM, Hong Kong, an extended version appeared in IEEE Transactions on Mobile Computing, 3(4).

[11] Shalli Rani et al, (2013) "EEICCP-Energy efficient protocol for wireless sensor network", Wireless sensor network, 5,127-136, scientific research.

[12] Amar shah, Kirti Patel, Prof. Pinal Patel, "A Survey of the Optimization of clustering techniques in Wireless sensor network", International journal of engineering development and research ISSN: 2321-9939

[13] S. Thiripura Sundari, P. Maheswara Venkatesh, Dr. A. Sivanantharaja, (2013) "A Novel Protocol for Energy Efficient Clustering for Heterogeneous Wireless Sensor Networks", International Journal of Scientific \& Engineering Research, Volume 4, Issue 8, ISSN 2229-5518

\section{Authors}

Monika G. Raghatate received Bachelor of Engineering degree in Computer Engineering from Bapurao Deshmukh college of engineering, Sewagram, Wardha, India. Presently, She is pursuing her M.Tech. In Computer Science and Engineering from St. Vincent Pallotti College of engineering and technology, Nagpur, India. Her research interest includes Wireless sensor network, Mobile Ad-hoc network, artificial neural network and web Technologies.

Prof. Dipak W. Wajgi received Bachelor of Engineering degree in Computer Technology from Kavi Kulguru Institute of Technology and Science, Ramtek, Nagpur, India. And M.Tech in Computer Science and Engineering from Ramdeobaba College of Engineering and Management, Nagpur, India. He has around thirteen years of teaching experience in engineering colleges. His research interest includes image processing, Wireless sensor network, pattern recognition, and artificial neural network. Presently, he is Assistant

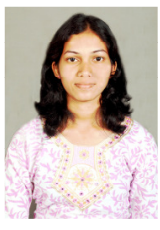
Professor in Department of Computer Engineering at St. Vincent Pallotti College of engineering and technology, Nagpur,India.

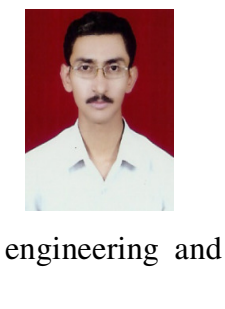

